SLAC-PUB-10200

October 2003

\title{
Light-Front Hadron Dynamics and AdS/CFT Correspondence*
}

\author{
Stanley J. Brodsky ${ }^{\dagger}$ \\ Stanford Linear Accelerator Center, \\ Stanford University, Stanford, California 94309, USA \\ Guy F. de Téramond $\ddagger$ \\ Universidad de Costa Rica, San José, Costa Rica
}

\begin{abstract}
A remarkable consequence of the AdS/CFT correspondence is the nonperturbative derivation of dimensional counting rules for hard scattering processes. Using string/gauge duality we derive the QCD power behavior of light-front Fock-state hadronic wavefunctions for hard scattering in the large- $r$ region of the AdS space from the conformal isometries which determine the scaling of string states as we approach the boundary from the interior of AdS space. The nonperturbative scaling results are obtained for spin-zero and spin- $\frac{1}{2}$ hadrons and are extended to include the orbital angular momentum dependence of the constituents in the light-front Fock-expansion. The correspondence with string states is considered for hadronic states of arbitrary orbital angular momentum for a given hadron of spin $\leq 2$. We examine the implications of the color configuration of hadronic Fock-states for the QCD structure of scattering amplitudes at large $N_{C}$. Quark interchange amplitudes emerge as the dominant large $N_{C}$ scattering mechanisms for conformal QCD.
\end{abstract}

\footnotetext{
*Work partially supported by the Department of Energy, contract DE-AC03-76SF00515.

${ }^{\dagger}$ E-mail: sjbth@slac.stanford.edu

${ }^{\ddagger}$ E-mail: gdt@asterix.crnet.cr
} 
Since the early identification of large $N_{C}$ conformal QCD with the topological expansion of a string [1], the connection between string theories and the large- $N_{C}$ limit of field theories has drawn major attention. However, string theory is not consistent in a four dimensional flat space, and at least one extra dimension has to be introduced [2. It is only recently that a precise correspondence has been established between quantum field theories and string/M-theory on Anti-de Sitter spaces $(\mathrm{AdS})$ 3], where strings live on the curved geometry of the AdS space and the observables of the conformal field theory are defined in the boundary of AdS. The partition function of the AdS theory is identified with the generating functional of the boundary conformal gauge field theory, and correlation functions are computed in the boundary of AdS where the quantum field theory operators are inserted [4, [5]. The AdS/CFT conjecture is indeed a concrete realization of the holographic principle 6, 7]. From the point of view of string theory/field theory duality, excitations near the boundary of the conformal AdS space correspond to states in the field theory. Hardscattering interactions occur in the large- $r$ region of the AdS space, so effectively we require the 't Hooft parameter, the product of the string coupling and the number of colors $g_{s} N_{C}$, to be sufficiently large so that the AdS radius $R=\left(4 \pi g_{s} N_{C}\right)^{1 / 4} \alpha^{1 / 2}$, with $\alpha^{\prime}$ the string scale, is also large. We can map the string states degrees of freedom to the QCD degrees of freedom at the boundary of the AdS space [8].

A remarkable consequence of the AdS/CFT correspondence is the derivation 9] of dimensional counting rules for the leading power-law fall-off of hard exclusive processes [10, 11]. The derivation from supergravity/string theory in a $A d S_{5} \times X$ background does not rely on perturbation theory, and thus is more general than perturbative QCD analysis [12. Classical QCD with massless quarks is conformal. Corrections from nonconformal effects in QCD due to the inverse logarithmic running of the coupling are moderate in the ultraviolet region. Theoretical [13, 14, 15] and phenomenological [16, 17] evidence is now accumulating that the QCD coupling becomes constant at small virtuality; i.e., $\alpha_{s}\left(Q^{2}\right)$ develops an infrared fixed point. Indeed, QCD appears to be a nearly-conformal theory in the momentum regimes accessible to experiment. The amplitudes of confining gauge theories with superstring duals, in addition to the power-law hard dependence at large momentum transfer, have Regge behavior at small angles for different kinematic domains [9]. The String/Gauge du- 
ality for QCD in four dimensions can also be obtained from M-theory in a specific Black Hole deformation of $A d S^{7} \times S^{4}$ [18] with Regge behavior in the near-forward limit [19]. The AdS/CFT correspondence for gauge theories with dual strings has been used to describe deep inelastic scattering [20], and the derivation of scaling laws in hadronic processes has been considered for exclusive processes, form factors and structure functions [21].

In this paper counting rules for light-front wavefunctions, the probability amplitudes which relate the constituents degrees of freedom with the asymptotic hadronic states, are obtained from the AdS/CFT correspondence for large momentum transfer processes as we approach the boundary of the AdS space. The nonperturbative scaling results are obtained for spin-zero and spin- $\frac{1}{2}$ hadrons. We are indeed limited to string states dual to hadrons of spin $0, \frac{1}{2}, 1, \frac{3}{2}$ and 2 , since in string theory there are only fields of spin $\leq 2$, and consequently all fields with higher spin have masses of order of the string scale $\left(\alpha^{\prime}\right)^{-1 / 2}$. The counting rules for light-front wavefunctions are also extended to include the orbital angular momentum dependence of the constituents by computing the corresponding scaling dimension from the conformal algebra of the generators and their action on the field operators. We also discuss the AdS/CFT string/gauge theory duality for Fock-states with orbital angular momentum by examining the Kaluza-Klein (KK) modes in the compact manifolds required to match the 10-dimensional critical-strings. Our goal is to use the AdS/CFT correspondence to constrain the form of the light-front wavefunctions of hadrons in QCD in the phenomenologically interesting near-conformal regime. Consequently we will evaluate the dual string theory at finite 't Hooft coupling $g_{s} N_{C}$ and well defined values of $\lambda R$, where $\lambda$ is the characteristic energy of a KK excitation.

It is well known that the quark interchange amplitudes for fixed-angle exclusive processes constitute a dominant mechanism in the large 't Hooft limit [1] $N_{C} \rightarrow \infty$, keeping the product $g_{Q C D}^{2}(\mu) N_{C}$, with $g_{Q C D}^{2} \sim g_{s}$, large but fixed at some given scale $\mu$. The quark interchange amplitudes are planar Feynman diagrams and are leading amplitudes in a $1 / N_{C}$ expansion, with other non-planar contributions being suppressed by powers of $1 / N_{C}$. This result is related to hadronic duality [23], since in the large $N_{C}$ limit quark-interchange amplitudes are topologically equivalent to duality diagrams which keep track of the flow of quark quantum numbers; they rep- 
resent string world-sheets with the quark lines defining the boundaries. There is also a set of graphs corresponding to multiple gluon exchange, where the gluons are connected to the same quark at each hadron and are clearly planar. However, planarity is not sufficient to ensure that a diagram is leading in the $1 / N_{C}$ expansion. Multiple gluon exchange planar diagrams are also considered, and it is shown that they are suppressed relative to quark interchange.

The quark interchange amplitude for meson-meson scattering scales as $1 / N_{C}$ while other contributions scale faster as $N_{C} \rightarrow \infty$. The case for baryons is more complicated since the baryons have $N_{C}$ quarks in their valence state, and the usual description of baryon elastic scattering 22 gives a result which diverges linearly with $N_{C}$ for quark exchange amplitudes. It will be shown below that if proper account is taken of the color configuration in the normalization of the Fock components, the antisymmetrization properties of the $S U\left(N_{C}\right)$ baryon wave function leads to the same $N_{C}$ counting rules for baryon and meson states. This is quite satisfactory. For example, the sum of the photon attachments in a form factor should give the total charge independent of $N_{C}$, regardless that we are describing a meson or a baryon. The string/field theory duality predictions for hard scattering correspond precisely to the $N_{C} \rightarrow \infty$ limit, which is also the large $g_{S} N_{C}$ limit. For large transverse momentum the relevant scale is $\mu \sim Q$, and quark exchange interactions occur in the large- $r$ conformal region of the $A d S$ space.

In QCD the Hilbert space is constructed in terms of a complete Fock basis of non interacting constituents at fixed light-front time, where the amplitudes are $n$-parton light-front wave functions $\psi_{n / h}$ corresponding to the expansion of color singlet hadron states

$$
\left|\Psi_{h}\right\rangle=\sum_{n} \psi_{n / h}|n\rangle
$$

The light-front Fock-state wavefunction provides a frame-independent representation of relativistic composite systems in QCD at the amplitude level, in terms of quark and gluon degrees of freedom which carry the symmetries within the hadrons.

According to the AdS/CFT correspondence, a quantum gravity theory in AdS space defines a conformal field theory on its boundary [3], where a string state is related with its corresponding renormalized four-dimensional quantum field operator 4, 5]. We thus expect that for large values of the 't Hooft parameter $g_{s} N_{C}$, 
near-conformal QCD has an effective dual string description. We will show that the scaling properties of the $n$-parton light-front wave functions are obtained from the string/gauge theory duality, and their power-law dependence follows from the conformal dimension of the corresponding string state dual to hadron states. The zero-mode states of strings can be treated classically, whereas the higher Fock components of Eq. (11) are a manifestation of the quantum fluctuations of QCD. Indeed, Hilbert space is defined in the boundary theory and not within the interior of AdS space. Since string theory predicts the scaling behavior of string states corresponding to different numbers of constituents, we can apply this information to the higher Fock components of physical hadrons and their corresponding interpolating operators. The correspondence is particularly useful for exclusive reactions at large momentum which are controlled by Fock components with a minimum number of constituents. We will also examine the correspondence of hadron Fock states with non-zero orbital angular momentum and string states with Kaluza-Klein excitations in the compact space $X$. In this case the discussion is more complex, since the dimensions of the states are in general not protected from acquiring large values at strong coupling, and considerations about the chirality properties of states are important.

To conserve Poincaré invariance in four dimensional Minkowski space the metric should have the form

$$
d s^{2}=w(z)^{2}\left[d x^{2}-d z^{2}\right]
$$

where $d x^{2}=\eta_{\mu \nu} d x^{\mu} d x^{\nu}$. Reparametrization invariance allows us to factor out the warp factor $w(z)^{2}$. The coordinates $x^{\mu}$ are Minkowski spacetime variables and $z$ labels the new spatial dimension. If the four-dimensional gauge theory is conformal, it is invariant under the transformation $x \rightarrow \rho x$. If $z \rightarrow \rho z$ is also an isometry of (2), then $w(z)=R / z$ and the space is a five-dimensional Anti-de Sitter space, $A d S_{5}$, and the conformal group $S O(2,4)$ in Minkowski space is also the group of symmetries of $A d S_{5}$. The value $z=0$ corresponds to the four-dimensional boundary where the gauge theory is defined. Introducing a radial coordinate $r=R^{2} / z$ we can express the $A d S_{5}$ metric (2) as

$$
d s^{2}=\frac{r^{2}}{R^{2}}\left(d t^{2}-d \vec{x}^{2}\right)-\frac{R^{2}}{r^{2}} d r^{2} .
$$

If the conformal gauge theory is dual to a critical string, a superstring theory in 10 dimensions, spacetime is the product space $A d S_{5}$ with a five-dimensional manifold $X$. 
In the original Maldacena conjecture [3] a correspondence was established between Type IIB string - a closed string with left-right asymmetric vibrational patterns compactified on $A d S_{5} \times S^{5}$, and $\mathcal{N}=4$ large $N_{C}$ superconformal Yang-Mills theory at the boundary, with the solution $R=\left(4 \pi g_{s} N_{C}\right)^{1 / 4} \alpha^{1 / 2}$ for the radius of AdS and the radius of the five-sphere. The theory with maximal supersymmetry corresponds to the sphere.

The observables of the gauge theory are defined at the boundary of the AdS space. The AdS metric implies that a distance $L_{\text {local }}$, measured in the local inertial coordinates in the full space shrinks by a warp factor as one approaches the AdS boundary. Thus the length at large- $r$ as observed in Minkowski space is

$$
L_{r}=\left(\frac{R}{r}\right) L_{\text {local }}
$$

Consequently, a local scattering state in the bulk with local momentum $Q_{\text {local }}$ as determined in the inertial frame is shifted to the ultraviolet as measured by an observer in Minkowski space

$$
Q_{r}=\left(\frac{r}{R}\right) Q_{\text {local }}
$$

Since the characteristic energy scale in the ten-dimensional space is $Q_{\text {local }} \sim R^{-1}$, it follows that $r \sim Q R^{2}$. Thus large $Q$ pointlike hard scattering processes occur in the large-r conformal region of the AdS space.

The geometry at small values of $r$ and the form of the compact space $X$ depend on the nature of the gauge theory and the mechanisms of conformal symmetry breaking. QCD is a nearly conformal theory in the ultraviolet region and a confining theory in the infrared with a mass gap corresponding to the scale $\Lambda_{o}$ of the lightest glueball scale. We thus expect that a non-conformal metric will be manifest at $r \sim r_{o}=\Lambda_{o} R^{2}$. However, we are interested in probing the wavefunction at large transverse momentum of order $Q \sim r / R^{2} \gg \Lambda_{o}$. The interaction occurs in the $r \sim Q R^{2} \gg r_{o}$ region where the conformal geometry (3) is valid and where the specific form of the compact space $X$ is not relevant [9].

Coordinates on $A d S_{5}$ are $x^{\mu}$ and $r$, and coordinates in the compact space are labelled $y^{a}$. According to the AdS/CFT correspondence we expect hadronic states at the boundary to have dual scattering states in the bulk. Consider first a spin-zero hadron state with four momentum $P^{\mu}$ in four-dimensional Minkowski space. The 
physical hadron in the boundary theory is expected to be dual to a string state represented by a 10-dimensional wavefunction $\Phi$, which is identified with a spinless dilaton field [9] satisfying the Laplace equation in ten-dimensional curved spacetime. For large values of $r \gg r_{o}$ spacetime geometry is the product space $A d S \times X$. The field $\Phi$ is expanded in terms of a complete set of normalized eigenfunctions $\phi_{l}$ of the fivedimensional Laplace operator, with proper boundary conditions on the compact space $X$ and eigenvalues $\lambda_{l}^{2}$. We write $\Phi(x, r, y)=\sum_{l} \Psi_{l}(x, r) \phi_{l}(y)$. From the point of view of ten-dimensional physics $\Phi$ is a string zero-mode of the ten-dimensional Laplace equation. However, we can still have a complete set of excited states corresponding to the Kaluza-Klein modes yet having zero modes in ten dimensions. Nonzero eigenvalues on $X$ are of the order of the inverse of the radius of the compact space $X$. For each eigenvalue $\lambda_{l}$ in the compact manifold $X$, the field $\Psi$ satisfies a $\mathrm{d}+1$ dimensional scalar equation in the warped geometry of $A d S_{d+1}$ space with the linearly independent solutions

$$
\Psi(x, r)=C e^{-i P \cdot x} r^{-\frac{d}{2}}\left\{\begin{array}{c}
J_{\alpha}\left(\frac{\mathcal{M} R^{2}}{r}\right) \\
N_{\alpha}\left(\frac{\mathcal{M} R^{2}}{r}\right)
\end{array},\right.
$$

where $\alpha^{2}=(d / 2)^{2}+(\lambda R)^{2}$ and $\mathcal{M}$ is the hadronic invariant mass, $P_{\mu} P^{\mu}=\mathcal{M}^{2}$. The dominant contribution at large- $r$ is $\Psi(r) \sim r^{-\Delta}$, where

$$
\Delta=\frac{1}{2}\left(d+\sqrt{d^{2}+4 \lambda^{2} R^{2}}\right)
$$

is the conformal dimension for a scalar field in $A d S_{d+1}$. At large relative transverse momentum, $Q \sim r / R^{2}, \Psi$ has the scaling behavior $\Psi(Q) \sim Q^{-\Delta}$ in the large- $r$ region of AdS space. The limiting values of the operators in the bulk are the Heisenberg field operators of the gauge field theory [3] specified by the boundary conditions [4, 5]

$$
\lim _{r \rightarrow \infty} \Psi(x, r)=r^{-\Delta} \Psi_{o}(x),
$$

where $\Psi_{o}(x)$ is the corresponding renormalized operator of the quantum field theory [26]. We shall first establish the correspondence with the QCD partonic degrees of freedom in the Fock expansion of hadronic quantum states in the AdS boundary for the case $\lambda=0$, corresponding to a zero mode in $X$ space. We will return to the excited modes on $X$, when discussing the extension of our results to include the orbital angular momentum of the Fock components. 
The basic constituents in QCD appear from the light-front quantization of the excitations of the dynamical fields, expanded in terms of creation and annihilation operators on the transverse plane with coordinates $x^{-}=z-c t$ and $\vec{x}_{\perp}$ at $\tau=$ $z+c t=0$. The expansion of bound state hadronic systems in terms of Fock states provides an exact representation of the local matrix elements used for calculating form factors, distribution amplitudes, and generalized parton distributions 24]. In terms of the hadron four-momentum $P=\left(P^{+}, P^{-}, \vec{P}_{\perp}\right)$ with $P^{ \pm}=P^{0} \pm P^{3}$, the light-front frame independent Hamiltonian for a hadronic composite system $H_{L C}^{Q C D}=P_{\mu} P^{\mu}=$ $P^{-} P^{+}-\vec{P}_{\perp}^{2}$, has eigenvalues given in terms of the eigenmass $\mathcal{M}$ squared corresponding to the mass spectrum of the color-singlet states in QCD, $H_{L C}^{Q C D}\left|\Psi_{h}\right\rangle=\mathcal{M}_{h}^{2}\left|\Psi_{h}\right\rangle$. The hadron state $\left|\Psi_{h}\right\rangle$ is expanded in a Fock-state complete basis of non-interacting $n$ particle states $|n\rangle$ with an infinite number of components

$$
\left|\Psi_{h}\left(P^{+}, \vec{P}_{\perp}\right)\right\rangle=\sum_{n, \lambda_{i}} \int\left[d x_{i} d^{2} \vec{k}_{\perp i}\right] \psi_{n / h}\left(x_{i}, \vec{k}_{\perp i}, \lambda_{i}\right)\left|n: x_{i} P^{+}, x_{i} \vec{P}_{\perp}+\vec{k}_{\perp i}, \lambda_{i}\right\rangle,
$$

where the coefficients of the light-front Fock expansion

$$
\psi_{n / h}\left(x_{i}, \vec{k}_{\perp i}, \lambda_{i}\right)=\left\langle n: x_{i}, \vec{k}_{\perp i}, \lambda_{i} \mid \psi_{h}\right\rangle
$$

depend only on the relative partonic coordinates, the longitudinal momentum fraction $x_{i}=k_{i}^{+} / P^{+}, \quad \sum_{i=1}^{n} x_{i}=1$, the relative transverse momentum $\vec{k}_{\perp i}, \quad \sum_{i=1}^{n} \vec{k}_{\perp i}=\overrightarrow{0}$, and $\lambda_{i}$, the projection of the constituents' spin along the $z$ direction. The amplitudes $\psi_{n / h}$ represent the probability amplitudes to find on-mass-shell constituents $i$ with momentum $x_{i} P^{+}$and $x_{i} \vec{P}_{\perp}+\vec{k}_{\perp i}$ and spin projection $\lambda_{i}$ in the hadron $h$. The measure of the constituents' phase-space momentum integration $\left[d x_{i} d^{2} \vec{k}_{\perp i}\right]$ depends on the normalization chosen. The complete basis of Fock-states $|n\rangle$ is constructed by applying free-field creation operators to the vacuum state $|0\rangle$ which has no particle content, $P^{+}|0\rangle=0, \vec{P}_{\perp}|0\rangle=0$. Since all the quanta have positive $k^{+}$, the vacuum state is unique and equal to the nonperturbative vacuum. A one-particle state is defined by $|q\rangle=\sqrt{2 q^{+}} a^{\dagger}(q)|0\rangle$ so that its normalization has the Lorentz invariant form $\left\langle q \mid q^{\prime}\right\rangle=2 q^{+}(2 \pi)^{3} \delta\left(q^{+}-q^{\prime+}\right) \delta^{(2)}\left(\vec{q}_{\perp}-\vec{q}_{\perp}^{\prime}\right)$. The measure of the phase space integration is defined by

$$
\left[d x_{i} d^{2} \vec{k}_{\perp i}\right]=\left(16 \pi^{3}\right) \delta\left(1-\sum_{j=1}^{n} x_{j}\right) \delta^{(2)}\left(\sum_{\ell=1}^{n} \vec{k}_{\perp \ell}\right) \prod_{i=1}^{n} \frac{d x_{i}}{x_{i}} \frac{d^{2} \vec{k}_{\perp i}}{16 \pi^{3}}
$$


and a normalized hadronic state $\langle\psi \mid \psi\rangle=1$, can be expressed as a sum of overlap integrals of light-front wavefunctions

$$
\sum_{n} \int\left[d x_{i} d^{2} \vec{k}_{\perp i}\right]\left|\psi_{n / h}\left(x_{i}, \vec{k}_{\perp i}, \lambda_{i}\right)\right|^{2}=1
$$

If the light-front wavefunctions do not fall quickly enough, infinities appear in the unitarity sum given by (12). To avoid this problem we truncate the Fock-states for light-front transverse momenta $\vec{k}_{\perp}^{2}$ above an ultraviolet scale $\Lambda^{2}[12$. Certainly the introduction of a cutoff explicitly breaks the conformal invariance of the theory. When the coupling falls as an inverse logarithm, the anomalous dimensions of an operator yield logarithmic corrections to the scattering amplitudes; if the coupling is constant the anomalous dimensions lead to power-law corrections [25].

The cutoff is usually taken in the limit $\Lambda \rightarrow \infty$ when performing calculations. In practice, the cutoff has no effect on the results provided that $\Lambda$ is much greater than all mass scales, so calculations are carried out with a finite cutoff by defining the wavefunctions, masses and couplings at the scale $\Lambda$. In a field theory where only a single scale $Q$ is relevant, it is natural to take the cutoff $\Lambda \sim Q$ and redefine the basic parameters and wavefunctions at the scale $Q$. Discarding Fock states with transverse momenta above $\Lambda$ and taking the effective cutoff $\Lambda$ as the scale $Q$ for large values of the momentum transfer, we can determine the large $\vec{k}_{\perp}^{2}$ momentum dependence of the light front amplitudes since we know the conformal behavior of the bulk state as we approach the boundary from the interior of AdS space. The correspondence at large- $r$ follows from the conformal isometry which determines the scaling of the string states.

To establish the correspondence between the hadron state and its dual string state and determine the precise counting rule of each Fock component, consider an operator $\Psi_{h}^{(n)}$ which creates an $n$-partonic state by applying n-times $a^{\dagger}\left(k^{+}, \vec{k}_{\perp}\right)$ to the vacuum state, creating $n$-constituent individual states with plus momentum $k^{+}$and transverse momentum $\vec{k}_{\perp}$. Each higher $n$-parton projection in the Fock expansion (9) corresponds to the higher-twist interpolating operator $\Psi_{h}^{(n)}$. Integrating over the relative coordinates $x_{i}$ and $\vec{k}_{\perp i}$ for each constituent using the expression for the phase space (11), we find the ultraviolet behavior of $\Psi_{h}^{(n)}$

$$
\Psi_{h}^{(n)}(Q) \sim \int^{Q^{2}}\left[d^{2} \vec{k}_{\perp}\right]^{n-1}\left[a^{\dagger}\left(\vec{k}_{\perp}\right)\right]^{n} \psi_{n / h}\left(\vec{k}_{\perp}\right)
$$


where the operator $a^{\dagger}\left(\vec{k}_{\perp}\right)$ scales as $1 / k_{\perp}$ at large $\vec{k}_{\perp}^{2}$. We obtain the scaling behavior of each hadronic interpolating field $\Psi^{(n)}$ from (6) replacing the hadronic invariant mass $\mathcal{M}$ with the off-shell invariant mass of the $n$-partonic state

$$
\mathcal{M}_{n}^{2}=\sum_{i=1}^{n} k_{i}^{\mu} k_{i \mu}=\sum_{i=1}^{n} \frac{k_{\perp i}^{2}+m_{i}^{2}}{x_{i}}
$$

since Fock-states are eigenstates of the free light-front Hamiltonian, and $\mathcal{M}_{n}$ is computed from the sum of the non-interacting contributions. Near the boundary of AdS space $\Psi^{(n)} \sim Q^{-\Delta_{n}}$, since $\mathcal{M}_{n}$ is a hadronic scale. The behavior of the light-front wavefunctions for large $\vec{k}_{\perp}^{2}$ then follows immediately

$$
\psi_{n / h}\left(k_{\perp}\right) \sim\left(k_{\perp}\right)^{\Delta_{n}-n}\left[\frac{1}{k_{\perp}^{2}}\right]^{\Delta_{n}-1} .
$$

The conformal dimension $\Delta_{n}$ is nominally the number of constituents since each interpolating fermion and gauge field operator has a minimum twist (dimension minus spin) of one. We thus identify $\Delta_{n}=n+2 \delta_{n}$ where $\delta_{n}$ represents the effect of the anomalous dimension of the higher-twist interpolating operators. Thus the light-front Fock state has the leading power behavior

$$
\psi_{n / h}\left(\vec{k}_{\perp}\right) \rightarrow\left(\frac{1}{\vec{k}_{\perp}^{2}}\right)^{n+\delta_{n}-1}
$$

Notice that the light-front amplitude $\psi_{n / h}$ does not scale as its mass dimension $M^{-n+1}$.

Although we have derived the scaling behavior of light-front amplitudes for a spinless hadron, identical scaling is obtained for a spin- $\frac{1}{2}$ hadron corresponding to a massless dilatino state $\chi$ in the bulk that obeys the massless Dirac equation in the 10-dimensional curved geometry of spacetime. In the large- $r$ conformal region the dilatino field is expanded in terms of eigenfunctions $\eta_{l}$ of the Dirac operator in compact space $X$ with eigenvalues $\lambda_{l}$. Specifically $\chi(x, r, y)=\sum_{l} \Psi_{l}(x, r) \eta_{l}(y)$, where $\Psi$ is a spinor field defined in a conformal $\mathrm{d}+1$ space and $\eta$ is a compact manifold spinor. For each eigenvalue $\lambda_{l}$ on $X, \Psi_{l}$ obeys a d +1 dimensional Dirac equation in the warped $A d S_{d+1}$ space with the solution [27]

$$
\Psi(x, r)=C e^{-i P \cdot x} r^{-\frac{d+1}{2}}\left[J_{\alpha}\left(\frac{\mathcal{M} R^{2}}{r}\right) \mu_{+}(P)+J_{\alpha+1}\left(\frac{\mathcal{M} R^{2}}{r}\right) \mu_{-}(P)\right],
$$


where $\alpha=\lambda R-\frac{1}{2}, \mathcal{M}=\sqrt{P_{\mu} P^{\mu}}$ and $\hat{\Gamma} \mu_{ \pm}= \pm \mu_{ \pm}$. If $d$ is even $\hat{\Gamma}=i \Gamma_{1} \ldots \Gamma_{d}$ is the $d$-dimensional chirality operator and the spinors in Eq. (17) are chiral. For $A d S_{5}, \hat{\Gamma}$ is the four dimensional chirality operator $\gamma_{5}$. The dominant contribution at large- $r$ also scales as $r^{-\Delta}$ where

$$
\Delta=\frac{d}{2}+|\lambda R|
$$

is the conformal dimension for a spinor field in $A d S_{d+1}$. The scaling is not changed by the spinors since they are boundary spinors.

In QCD each Fock state is an eigenfunction of the total angular momentum projection $J^{z}=\sum_{i=1}^{n} s_{i}^{z}+\sum_{i=1}^{n-1} l_{i}^{z}$, where the sum over the spin $s_{i}^{z}$ corresponds to the intrinsic spins of the n-constituent Fock states, and the sum over the $n-1$ orbital angular momenta $l_{i}^{z}=-i\left(k_{i}^{1} \frac{\partial}{\partial k_{i}^{2}}-k_{i}^{2} \frac{\partial}{\partial k_{i}^{1}}\right)$ excludes the contribution from the motion of the center-of-mass [28]. Parton orbital angular momentum components in the light-front expansion of hadronic states are essential in order to describe hadron spin-flip amplitudes in QCD, such as the $\ell=1$ proton $|u u d\rangle$ state which is required to have a non-zero Pauli form factor.

In $d$-dimensional Minkowski space it is simple to extend our previous scaling results to obtain the power fall-off behavior at high relative transfer momentum of light-front wave functions including orbital angular momentum. The derivation follows immediately from the algebra of the generators of the conformal group $S O(2, d)$. In particular, the generator of scaling transformations $D$ and the generator of translations $P_{\mu}$ obey the commutation relations $\left[D, P_{\mu}\right]=-i P_{\mu}$. Consider a state with orbital angular momentum $\ell, \Psi_{\ell} \sim p^{\ell} \Psi$, where the interpolating field $\Psi$ is an eigenfunction of the scaling operator $D$ with eigenvalue $-i \Delta[29]$

$$
[D, \Psi]=i\left(-\Delta+x^{\mu} \partial_{\mu}\right) \Psi
$$

Using the commutation relation of the operator $D$ with $P_{\mu}$ as written above, we obtain the scaling dimension $\Delta_{\ell}$ of the field $\Psi_{\ell}$ representing a state with orbital angular momentum $\ell$

$$
\left[D, \Psi_{\ell}\right]=i\left(-\Delta_{\ell}+x^{\mu} \partial_{\mu}\right) \Psi_{\ell}
$$

with $\Delta_{\ell}=\Delta+\ell$. The dimensional scaling law for the light-front wavefunctions at large $Q$ including constituent orbital angular momentum $\ell$ follows directly from the 
identification $\Delta_{n} \rightarrow \Delta_{n, \ell}=n+2 \delta_{n}+\ell$ in (15)

$$
\psi_{n / h}\left(\vec{k}_{\perp}\right) \rightarrow\left(k_{\perp}\right)^{\ell}\left[\frac{1}{\vec{k}_{\perp}^{2}}\right]^{n+\delta_{n}+\ell-1} .
$$

Since $O(d+1,1)$ the isometry group of $A d S_{d+1}$ in $d+1$ dimensions, acts as the conformal group $S O(2, d)$ in d-dimensional Minkowski space [30], it is natural to ask if a correspondence can be established between a string moving in AdS space and the QCD Fock-states with orbital angular momentum. According to the AdS/CFT duality, we would expect that all the states of the hadronic expansion in a complete Fock-basis spanning all the Hilbert space of the boundary theory are matched with the string degrees of freedom in a one-to-one correspondence. We know from string theory that the correspondence could not be established for spin greater than two, and a Fock-state can have high orbital angular momentum. However, the QCD eigenstate itself has no large spin, since the $J_{z}$ component of each Fock-state is identical to that of the hadron itself. If the sum of orbital components $l_{i}^{z}$ is large, it is compensated by the sum of the constituents' spin $s_{i}^{z}$ for each of the $n-1$ orbital angular momentum states corresponding to a Fock-state with $n$ partons.

From (71) or (18) it is clear that only if the compact space $X$ has dimensions of order $R$, would the product $\lambda R$ be independent of the 't Hooft coupling. The KK states should also be protected from quantum or string effects which potentially can give contributions of the order of the string scale to $\Delta$. As an example, for a string that lives on $M^{4} \times K$, the product of Minkowski spacetime and a six-dimensional compact space $K$, excited KK states are non-chiral [31]. Consequently, no mechanism can in this case prevent the excited eigenmodes from acquiring a very large mass from invariant mass terms at tree level in the Lagrangian. The non-chiral states acquire a mass given by the highest scale available, $\lambda \sim 1 / \sqrt{\alpha^{\prime}}$, and decouple from the theory according to the survival hypothesis 32 , leaving effectively the massless modes. If $(\lambda R)^{2} \sim\left(g_{s} N_{C}\right)^{1 / 2}$, we would expect from (7) or (18) the dimension of an excited KK state to grow as $\Delta \sim\left(g_{s} N_{C}\right)^{1 / 4}$ at large 't Hooft coupling. In the case of the supersymmetric Yang-Mills correspondence with Type IIB strings on $A d S_{5} \times S^{5}[3$, all Kaluza-Klein excited states transform in short supergravity multiplets and the radius of curvature of $S^{5}$ is also $R$. The associated dimensions are protected by the supersymmetry algebra [8]. 
Strings moving in the warped geometry of $A d S_{5} \times X$ spacetime involve both positive and negative chirality solutions as given by Eq. (17). In this case, the opposite chirality states have different dimensions and thus very different dependences on $r$; they do not pair to form an invariant mass term. Indeed only the positive chirality solution is dominant at large $r$, corresponding to a QCD state with proper near-conformal scaling at the AdS boundary. Contrary to strings on $M^{4} \times K$, the dimensions associated with $\mathrm{KK}$ modes for strings on $A d S_{5} \times X$ space are protected, i.e. the dimensions are independent of the 't Hooft coupling as long as the compact space has dimensions of order $R$.

Harmonic KK modes are eigenstates of the Laplacian on an N-sphere $S^{N}$ with eigenvalues $l(l+N-1)$, where $l$ is an integer. For $N=d+1$, the product $\lambda R$ is thus quantized according to $(\lambda R)^{2}=l(l+d)$. From (17) it follows that $\Delta_{l}=\Delta+l$, where $\Delta$ is the conformal dimension corresponding to a KK zero mode. The matching of states in the AdS/CFT correspondence is carried out near the boundary of AdS space $r=R^{2} / z$ with $z \sim 0$. We then can identify $l=\ell$.

The case for the spinor field is slightly more complex. The Dirac operator on an $\mathrm{N}$-sphere has eigenvalues [33] $\pm\left(l+\frac{N}{2}\right)$, where $l$ is a non negative integer $l=$ $0,1,2, \ldots$ Consequently $\lambda R$ obeys the quantization condition $\lambda R= \pm\left(l+\frac{d}{2}+\frac{1}{2}\right)$ for $N=d+1$, and the lowest KK Dirac mode on the $\mathrm{N}$-sphere has non-zero mass. From (18) we obtain for the conformal dimension of the spinor field $\Delta_{l}=d+l+\frac{1}{2}$, and thus the relation $\Delta_{l}=\Delta+l$ is also valid in this case. Here also $\Delta$ is the conformal dimension for $l=0$. We have thus established a correspondence of orbital angular momentum components for the Fock expansion of meson and baryon states with the corresponding KK modes in the compact space. Only 10-dimensional string excitations, or stringy excitations, have masses of order of the string scale $\left(\alpha^{\prime}\right)^{-1 / 2}$ and dimensions $\Delta \sim\left(g_{s} N_{C}\right)^{1 / 4}$.

To specify the angular momentum properties of hadronic states [34] in QCD, the orbital angular momentum component of the hadron wavefunction is constructed in terms of the $n-1$ transverse momenta of the components and has the general structure [35]: $\left(k_{1 \perp}^{ \pm}\right)^{\left|l_{z 1}\right|}\left(k_{2 \perp}^{ \pm}\right)^{\left|l_{z 2}\right|} \ldots\left(k_{(n-1) \perp}^{ \pm}\right)^{\left|l_{z(n-1)}\right|}$, with $k_{i \perp}^{ \pm}=k_{i}^{1} \pm i k_{i}^{2}$. In the regime of moderate value of the 't Hooft coupling we expect the anomalous dimension to be small to retain the near-conformal features of QCD phenomena. The phenomeno- 
logical success of dimensional counting rules suggest indeed that the effect of the anomalous dimensions is small. We thus write for the hard component of the lightfront wavefunction

$$
\psi_{n / h}\left(x_{i}, \vec{k}_{\perp i}, \lambda_{i}, l_{z i}\right) \sim \frac{\left(g_{s} N_{C}\right)^{\frac{1}{2}(n-1)}}{\sqrt{\mathcal{N}}_{C}} \prod_{i=1}^{n-1}\left(k_{i \perp}^{ \pm}\right)^{\left|l_{z i}\right|}\left[\frac{\Lambda_{o}}{\mathcal{M}^{2}-\sum_{i} \frac{\vec{k}_{\perp i}^{2}+m_{i}^{2}}{x_{i}}+\Lambda_{o}^{2}}\right]^{n+\left|l_{z}\right|-1}
$$

where $\Lambda_{o}$ represents the basic QCD mass scale and the normalization factor $\left(\mathcal{N}_{\mathcal{C}}\right)^{-1 / 2}$ depends on the color structure of each Fock state. For example for a valence meson state $\mathcal{N}_{C}=N_{C}$, for a valence baryon state $\mathcal{N}_{C}=N_{C}$ !, and for the lowest component glue state $\mathcal{N}_{C}=N_{C}^{2}$. The form (22) is compatible with the scaling properties predicted by the AdS/CFT correspondence (21) including orbital angular momentum [36].

Fixed-angle large transverse momentum exclusive collision processes in QCD take place in the large conformal region of AdS space. Let us review first the results for hard meson scattering in a theory with gauge symmetry $S U\left(N_{C}\right)$ for large $N_{C}$ [37. We use the 't Hooft double-line representation [1] of Feynman diagrams where a quark propagator is represented by a single-index line and a gluon propagator by two-index lines. To obtain the $1 / N_{C}$ expansion for a meson form factor $F$, we note that there is a factor of $N_{C}$ from a closed color quark loop where the photon is attached and a normalization factor of $1 / \sqrt{N}_{C}$ for each meson wave function and thus $F \propto N_{C}^{0}$, independent of $N_{C}$ as it should.

To compute the meson-meson scattering amplitude $M$ in the large $N_{C}$ limit, we include a factor of $N_{C}$ from a closed color quark loop from quark interchange and a factor of $1 / \sqrt{N}_{C}$ for the normalization of each meson wave function; thus $M_{Q I M} \propto$ $1 / N_{C}$. The counting rule is not changed at fixed $g_{Q C D}^{2} N_{C}$ by any number of ladder gluon exchanges between quarks within the same meson, as would result from the iteration of the equation of motion of the meson wavefunction. The evaluation of planar multiple gluon exchange diagrams is also simple. In the case of two-gluon exchange in meson-meson scattering, the index color-counting of the gluon exchange in terms of $q \bar{q}$ pairs gives an additional factor $N_{C}$ relative to the quark interchange diagram from the additional quark color loop, but each vertex has a factor $1 / \sqrt{N}_{C}$ and thus $M_{2 g} \propto 1 / N_{C}^{2}$. The $1 / N_{C}^{2}$ dependence is not modified by additional exchange of gluons, since each new gluon introduces two extra vertices and one additional closed 
color loop which cancels the $N_{C}$ factor, and the scaling is given by the wavefunction normalization; thus $M_{n g} \propto 1 / N_{C}^{2}$.

Baryons constitute a difficult problem in the large $N_{C}$ limit since they are represented by a totally antisymmetric color state with $N_{C}$ different quarks, and the number of quark lines in a given diagram grows with $N_{C}$. It is expected, however, that baryons will follow simple scaling laws in a $1 / N_{C}$ expansion. In his theory of baryons at large $N_{C}$, Witten uses graphical methods to describe the $n$-body force and introduces a bound state which consists of a large number of weakly interacting particles described in the Hartree approximation [22]. In the case under discussion, large transverse momentum hadron-hadron scattering, the problem is simplified, since all the $N_{C}$ constituents ( $N_{C}$ large but finite) change their collinear direction in the collision process and acquire high transverse momentum. Thus one requires the high$Q$ components of the hadronic wavefunction corresponding to large- $r$ values of AdS space given by (22), with the normalization factor $1 / \sqrt{N_{C}}$ ! for each baryon wave function. Then combinatorial factors for the quark loops are computed, giving the non-trivial $1 / N_{C}$ expansion with the usual index counting in the diagrams.

Consider first the $1 / N_{C}$ expansion for a baryon form factor $F$ for an $N_{C}$ component fully antisymmetric color singlet state $|B\rangle=\frac{1}{\sqrt{N_{C} !}} \epsilon_{i_{1}, i_{2}, \ldots, i_{N_{C}}}\left|q_{i_{1}} q_{i_{2}} \ldots q_{i_{N_{C}}}\right\rangle$, with $\langle B \mid B\rangle=1$. To evaluate the combinatoric factors it is useful to consider the color quark loop with the anti-color index from the effective remaining $N_{C}-1$ quarks in the baryon wavefunction. There is a factor $N_{C}$ from the closed color quark loop for the quark in the baryon attached to the high transverse momentum photon, times the factor $\left(N_{C}-1\right)$ ! to account for all the possible loops from the different permutations in the baryon wavefunction. Taking into account the normalization factor $\sqrt{N_{C}}$ ! for each baryon wavefunction we obtain $F \propto N_{C}^{0}$, independent of $N_{C}$.

For the baryon-baryon scattering amplitude $M$ there is a factor $N_{C}$ from a closed quark loop from quark interchange and a factor $\left[\left(N_{C}-1\right) !\right]^{2}$, which counts all the loops from the possible permutations from the two baryon wavefunctions. There is also a factor $\left(1 / \sqrt{N_{C} !}\right)^{4}$ from the baryon wavefunction normalization. Combining the different factors we obtain $M_{Q I M} \propto 1 / N_{C}$. The analysis of planar multiple gluon exchange diagrams is performed along the same lines giving an additional $1 / N_{C}$ factor compared to the dominant quark interchange amplitudes. Repeating the analysis 
for the $1 / N_{C}$ power counting for meson-baryon processes, or baryon-antibaryon scattering, we obtain identical results: the quark interchange amplitudes scale as $1 / N_{C}$, whereas multiple gluon amplitudes from planar graphs scale as $1 / N_{C}^{2}$. Non-planar contributions are naturally suppressed relative to the planar diagrams and could explain the absence of Landshoff pinch contributions [38] in the large transverse momentum fixed $t / s$ proton-proton scattering data, where each pair of quarks scatter independently by gluon exchange. These are nonplanar.

We are now in a position to write general expressions for transition amplitudes corresponding to the hard component of the light-front wavefunction (22) obtained from the AdS/CFT correspondence at large values of $g_{s} N_{C}$, for arbitrary number of components in the Fock expansion including orbital angular momentum. To compare with the quantum field theory results, the 't Hooft parameter $g_{s} N_{C}$ appearing in the light-front wavefunction (22) has to be scaled according $\sqrt{g_{s} N_{C}} \rightarrow g_{s} N_{C}$ [36]. The non-perturbative expression for the form factor at large transverse momentum is

$$
F\left(Q^{2}\right) \sim\left(g_{Q C D}^{2} N_{C}\right)^{n-1}\left[\frac{\Lambda_{o}}{Q}\right]^{2 n+\left|l_{z}\right|-2}, \quad g_{Q C D}^{2} \sim g_{s}
$$

where $n$ is the number of components in the Fock state and $\left|l_{z}\right|$ is the angular momentum component. The extra factor $\Lambda_{o}^{n-1}$ comes from the soft components of the wavefunction after integrating the exact Drell-Yan-West expression for the form factor [39] over $d^{2} \vec{k}_{\perp}^{n-1}$ phase space. For $l_{z}=0$ (23) agrees with the nominal counting perturbative QCD results. The equivalent expression for the hadronic scattering reaction $A+B \rightarrow C+D$ is obtained from the general structure of the quark interchange amplitude [4], including the appropriate number of integration loops

$$
M\left(Q^{2}\right)_{A+B \rightarrow C+D} \sim \frac{\left(g_{Q C D}^{2} N_{C}\right)^{\frac{1}{2}(n-2)}}{N_{C}}\left[\frac{\Lambda_{o}}{Q}\right]^{\left(n+\left|l_{z}\right|-4\right)}
$$

where $n$ is the total number of constituents entering the reaction, $n=n_{A}+n_{B}+$ $n_{C}+n_{D}$, and $g_{Q C D}^{2} \sim g_{s}$. The additional $\Lambda_{o}$ factor corresponds to the soft momentum components of the wavefunction from phase space loop integration.

In this paper we have shown how the scaling properties of the hadronic interpolating operator in the extended AdS/CFT space-time theory determines the scaling of light-front hadronic wavefunctions at high relative transverse momentum. The 
angular momentum dependence of the light-front wavefunctions also follows from the AdS/CFT correspondence mapping the Fock components into string states in $A d S \times X$ spacetime, and identifying the partonic states with orbital angular momentum with the Kaluza Klein eigenmodes in the compact space $X$. The scaling predictions agree with the perturbative QCD analysis given in Ref. [35, but here the analysis is performed at strong coupling without the use of perturbation theory. Quark interchange is the dominant mechanism at large momentum transfer in the $N_{C} \rightarrow \infty$ limit of QCD and consistent expressions are obtained for meson and baryon scattering at large transverse momentum. The near-conformal scaling properties of light-front wavefunctions lead to a number of predictions for QCD which are normally discussed in the context of perturbation theory, such as constituent counting scaling laws for the leading power fall-off of form factors and hard exclusive scattering amplitudes for QCD processes. The ratio of Pauli to Dirac baryon form factor has the nominal asymptotic form $F_{2}\left(Q^{2}\right) / F_{1}\left(Q^{2}\right) \sim 1 / Q^{2}$, modulo logarithmic corrections, in agreement with the perturbative results of Ref. 41]. Our analysis can also be extended to study the spin structure of scattering amplitudes at large transverse momentum and other processes which are dependent on the scaling and orbital angular momentum structure of light-front wavefunctions.

We thank Oleg Andreev, Joe Polchinski, Helen Quinn, Jörg Raufeisen, and Matt Strassler for helpful comments. 


\section{References}

[1] G. 't Hooft, Nucl. Phys. B 72, 461 (1974).

[2] A. M. Polyakov, Nucl. Phys. Proc. Suppl. 68, 1 (1998) arXiv:hep-th/9711002.

[3] J. M. Maldacena, Adv. Theor. Math. Phys. 2, 231 (1998) [Int. J. Theor. Phys. 38, 1113 (1999)] arXiv:hep-th/9711200.

[4] S. S. Gubser, I. R. Klebanov and A. M. Polyakov, Phys. Lett. B 428, 105 (1998) arXiv:hep-th/9802109.

[5] E. Witten, Adv. Theor. Math. Phys. 2, 253 (1998) arXiv:hep-th/9802150.

[6] G. 't Hooft, arXiv:gr-qc/9310026.

[7] L. Susskind, J. Math. Phys. 36, 6377 (1995) arXiv:hep-th/9409089.

[8] For a review of the correspondence between large $\mathrm{N}$ gauge theories and string theories see for example, O. Aharony, S. S. Gubser, J. M. Maldacena, H. Ooguri and Y. Oz, Phys. Rept. 323, 183 (2000) arXiv:hep-th/9905111.

[9] J. Polchinski and M. J. Strassler, Phys. Rev. Lett. 88, 031601 (2002) arXiv:hep-th/0109174.

[10] S. J. Brodsky and G. R. Farrar, Phys. Rev. Lett. 31, 1153 (1973); Phys. Rev. D 11, 1309 (1975).

[11] V. A. Matveev, R. M. Muradian and A. N. Tavkhelidze, Lett. Nuovo Cim. 7, 719 (1973).

[12] G. P. Lepage and S. J. Brodsky, Phys. Rev. D 22, 2157 (1980); Phys. Rev. Lett. 43, 545 (1979) [Erratum-ibid. 43, 1625 (1979)].

[13] L. von Smekal, R. Alkofer and A. Hauck, Phys. Rev. Lett. 79, 3591 (1997) arXiv:hep-ph/9705242.

[14] D. Zwanziger, arXiv:hep-ph/0303028 
[15] D. M. Howe and C. J. Maxwell, Phys. Lett. B 541, 129 (2002) arXiv:hep-ph/0204036; arXiv:hep-ph/0303163.

[16] A. C. Mattingly and P. M. Stevenson, Phys. Rev. D 49, 437 (1994) arXiv:hep-ph/9307266.

[17] S. J. Brodsky, S. Menke, C. Merino and J. Rathsman, Phys. Rev. D 67, 055008 (2003) arXiv:hep-ph/0212078.

[18] R. C. Brower and C. I. Tan, Nucl. Phys. B 662, 393 (2003) arXiv:hep-th/0207144.

[19] See also, H. Boschi-Filho and N. R. Braga, Phys. Lett. B 560, 232 (2003) arXiv:hep-th/0207071.

[20] J. Polchinski and M. J. Strassler, JHEP 0305, $012 \quad$ (2003) arXiv:hep-th/0209211.

[21] O. Andreev, Phys. Rev. D 67, 046001 (2003) arXiv:hep-th/0209256.

[22] E. Witten, Nucl. Phys. B 160, 57 (1979).

[23] H. Harari, Phys. Rev. Lett. 22 (1969) 562; J. L. Rosner, Phys. Rev. Lett. 22, 689 (1969); P.G.O. Freund, Nuovo Cimento Lett. 4, 147 (1970); R. Dolen, D. Horn and C. Schmid, Phys. Rev. 166, 1768 (1968).

[24] S. J. Brodsky, H. C. Pauli and S. S. Pinsky, Phys. Rept. 301, 299 (1998) arXiv:hep-ph/9705477.

[25] J. B. Kogut and L. Susskind, Phys. Rev. D 9, 697 (1974).

[26] In terms of the boundary conditions (8) the partition function in AdS space $Z_{A d S}$, is identified with the generating functional of the conformal field theory $Z_{C F T}$ on the boundary [4, 5]: $Z_{A d S}\left[\left.\Psi(x, r)\right|_{r \rightarrow \infty}=\Psi_{o}(x)\right]=Z_{C F T}\left[\Psi_{o}(x)\right]$.

[27] M. Henningson and K. Sfetsos, Phys. Lett. B 431, 63 (1998) arXiv:hep-th/9803251; W. Muck and K. S. Viswanathan, Phys. Rev. D 58, 106006 (1998) arXiv:hep-th/9805145. 
[28] S. J. Brodsky, D. S. Hwang, B. Q. Ma and I. Schmidt, Nucl. Phys. B 593, 311 (2001) arXiv:hep-th/0003082.

[29] G. Mack and A. Salam, Annals Phys. 53 (1969) 174.

[30] Technically, the symmetry group $S O(2, d)$ acts on $M_{d}$, the hypersurface at the boundary of $A d S_{d+1}$ space, $z=R^{2} / r=0$, a copy of d-dimensional Minkowski space plus a single point at $z=\infty$.

[31] E. Witten, Shelter Island II: Proceedings of the 1983 Shelter Island Conference on Quantum Field Theory and the Fundamental Problems of Physics, eds. R. Jackiw et al. (MIT Press, Cambridge, Mass.), 1985.

[32] H. Georgi, Nucl. Phys. B 156, 126 (1979).

[33] R. Camporesi and A. Higuchi, arXiv:gr-qc/9505009.

[34] V. A. Karmanov and A. V. Smirnov, Nucl. Phys. A 546, 691 (1992).

[35] X. D. Ji, F. Yuan and J. P. Ma, Phys. Rev. Lett. 90 (2003) 241601. These authors include the analysis of amplitude mixing under renormalization.

[36] The model wavefunction (22) does not include the soft component required for the convergence of the integrals when computing physical observables. Note also the fractional power in the 't Hooft parameter in the light-front wavefunction (22) that scales as $\left(g_{s} N_{C}\right)^{\frac{1}{2}(n-1)}$ instead of $\left(g_{s} N_{C}\right)^{(n-1)}$, as would be expected from PQCD counting. The fractional dependence comes from the replacement $g N \rightarrow \sqrt{g N}$ to obtain agreement with the string results [9, 21]. Similar dependence is found in the large $\mathrm{N}$ superconformal evaluation of a quark-antiquark potential. See: S. J. Rey and J. T. Yee, Eur. Phys. J. C 22, 379 (2001) arXiv:hep-th/9803001; J. M. Maldacena, Phys. Rev. Lett. 80, 4859 (1998) arXiv:hep-th/9803002.

[37] For a review of large N methods see for example, S. R. Coleman, SLAC-PUB2484 Presented at 1979 Int. School of Subnuclear Physics, Pointlike Structures Inside and Outside Hadrons, Erice, Italy, Jul 31-Aug 10, 1979; A. V. Manohar, arXiv:hep-ph/9802419. 
[38] P. V. Landshoff, Phys. Rev. D 10, 1024 (1974).

[39] S. D. Drell and T. M. Yan, Phys. Rev. Lett. 24, 181 (1970); G. B. West, Phys. Rev. Lett. 24, 1206 (1970).

[40] J. F. Gunion, S. J. Brodsky and R. Blankenbecler, Phys. Lett. B 39, 649 (1972), Phys. Rev. D8, 287 (1973).

[41] A. V. Belitsky, X. d. Ji and F. Yuan, Phys. Rev. Lett. 91, 092003 (2003) arXiv:hep-ph/0212351, and references therein. 